\title{
The structure of fractional spaces generated by multi-dimensional elliptic difference operator
}

\author{
Allaberen Ashyralyev ${ }^{1,2}$ and Sema Akturk ${ }^{3 *}$
}

\section{"Correspondence:}

semathakturk@gmail.com

${ }^{3}$ Department of Mathematics, Fatih

University, Buyukcekmece, Istanbul,

34500, Turkey

Full list of author information is

available at the end of the article

\begin{abstract}
In the present paper, the positivity of a multi-dimensional difference operator in the half-space is considered. The structure of fractional spaces generated by this operator is investigated. The equivalence of the norms of these fractional spaces and Hölder spaces is proved. In applications, the stability of difference schemes for elliptic differential equations is presented.
\end{abstract}

MSC: 35J25; 47E05; 34B27

Keywords: positive operator; fractional spaces; Green's function; Hölder spaces

\section{Introduction}

The role played by the positivity of differential and difference operators in Banach spaces in the study of the stability of solutions of boundary value problems for partial differential equations and the stability of difference schemes for partial differential equations, and of summation Fourier series converging in $C$-norm is well known (see [1-3]).

Recall that an operator $A$ densely defined in a Banach space $E$ with domain $D(A)$ is called positive in $E$, if its spectrum $\sigma_{A}$ lies in the interior of the sector of angle $\varphi, 0<\varphi<\pi$, symmetric with respect to the real axis, and, moreover, on the edges of this sector $S_{1}(\varphi)=$ $\left\{\rho e^{i \varphi}: 0 \leq \rho \leq \infty\right\}$ and $S_{2}(\varphi)=\left\{\rho e^{-i \varphi}: 0 \leq \rho \leq \infty\right\}$, and outside of the sector the resolvent $(A-\lambda I)^{-1}$ is subject to the bound (see [2])

$$
\left\|(A-\lambda I)^{-1}\right\|_{E \rightarrow E} \leq \frac{M}{1+|\lambda|},
$$

where $I$ is the identity operator. The infimum of all such angles $\varphi$ is called the spectral angle of the positive operator $A$ and is denoted by $\varphi(A)=\varphi(E, A)$.

Throughout the present work, we will indicate with $M$ positive constants which can be different from time to time and we are not interested in precise. We will write $M(\alpha, \beta, \ldots)$ to express the fact that the constant depends only on $\alpha, \beta, \ldots$.

The theory of differential and difference operators in Banach spaces and their related applications have been investigated by many scientists (see, for example, [4-30]).

Great progress has been made in the study of structure of interpolation spaces generated by positive operators from the view-point of the stability analysis of high-order accuracy

\section{Springer}


difference schemes for various partial differential equations. However, the positivity of difference operators and structure of fractional spaces generated by these operators in Banach spaces are not well investigated in general. Therefore, the investigation of structure of fractional spaces generated by positive difference operators in Banach spaces and its applications to the stability of difference schemes for various partial differential equations is an important subject.

For a positive operator $A$ in the Banach space $E$, let us introduce the fractional spaces $E_{\alpha}=E_{\alpha}(E, A)(0<\alpha<1)$ consisting of those $v \in E$ for which the norms

$$
\|v\|_{E_{\alpha}}=\sup _{\lambda>0} \lambda^{\alpha}\left\|A(A+\lambda I)^{-1} v\right\|_{E}
$$

are finite. Clearly, the positive operator commutes $A$ and its resolvent $(A-\lambda I)^{-1}$. By the definition of the norm in the fractional space $E_{\alpha}=E_{\alpha}(E, A)(0<\alpha<1)$, we get

$$
\left\|(A-\lambda I)^{-1}\right\|_{E_{\alpha} \rightarrow E_{\alpha}} \leq\left\|(A-\lambda I)^{-1}\right\|_{E \rightarrow E} .
$$

Thus, from the positivity of operator $A$ in the Banach space $E$ follows the positivity of this operator in fractional spaces $E_{\alpha}=E_{\alpha}(E, A)(0<\alpha<1)$.

In [8], Simirnitskii and Sobolevskii considered the difference operator $A_{1 h}^{x}$ which is an elliptic difference operator of an arbitrary high order of accuracy approximating the multidimensional elliptic operator $A_{1}^{x}=B^{x}+\delta I$.

Let us define the grid space $\mathbb{R}_{h}^{h-1}\left(0<h \leq h_{0}\right)$ as the set of all points of the Euclidean space $\mathbb{R}^{n-1}$ whose coordinates are given by

$$
x_{k}=s_{k} h, \quad s_{k}=0, \pm 1, \pm 2, \ldots, k=1, \ldots, n-1 .
$$

The number $h$ is called the step of the grid space. A function defined on $\mathbb{R}_{h}^{n-1}$ will be called a grid function. To the differential operator $B$ with constant coefficients of the form

$$
B^{x}=\sum_{|r|=2 m} b_{r} \frac{\partial^{r_{1}+\cdots+r_{n-1}}}{\partial_{x_{1}^{r_{1}} \cdots \partial_{x_{n-1}^{r_{n-1}}}}}
$$

we assign the difference operator

$$
B_{h}^{x}=h^{-m} \sum_{2 m \leq|s| \leq S} d_{s} \Delta_{1-}^{s_{1}} \Delta_{1+}^{s_{2}} \cdots \Delta_{(n-1)-}^{s_{2 n-3}} \Delta_{(n-1)+}^{s_{2 n-2}},
$$

which acts on functions defined on the entire space $\mathbb{R}_{h}^{n-1}$. Here, $s \in \mathbb{R}^{2(n-1)}$ is a vector with nonnegative integer coordinates,

$$
\Delta_{k \pm} f^{h}(x)= \pm\left(f^{h}\left(x \pm e_{k} h\right)-f^{h}(x)\right)
$$

and $e_{k}$ is the unit vector of the axis $x_{k}$.

An infinitely differentiable function of the continuous argument $y \in \mathbb{R}^{n-1}$ that is continuous and bounded together with all its derivatives is said to be smooth. Let $\varphi(y)$ be a 
smooth function on $\mathbb{R}^{n-1}$. Using the Taylor expansion of $\varphi(y)$, one can show that

$$
\sup _{x \in \mathbb{R}_{h}^{n-1}}\left|h^{-1} \Delta_{k \pm} \varphi(x)-\frac{\partial}{\partial y_{k}} \varphi(x)\right| \leq M(\varphi) h
$$

Here, the grid function $\varphi(x)$ and $\frac{\partial}{\partial y_{k}} \varphi(x)$ are the traces of the functions $\varphi(y)$ and $\frac{\partial}{\partial y k} \varphi(y)$, respectively. The last inequality means that the difference operator $h^{-1} \Delta_{k \pm}$ is a first-order approximation for the differential operator $\frac{\partial}{\partial y_{k}}$.

We say that the difference operator $B_{h}^{x}$ is a $\lambda$ th-order $(\lambda>0)$ approximation of the differential operator $B^{x}$ if the inequality

$$
\sup _{x \in R_{h}^{n-1}}\left|B_{h}^{x} \varphi(x)-B^{x} \varphi(x)\right| \leq M(\varphi) h^{\lambda}
$$

holds for any smooth function $\varphi(y)$. We shall assume that the operator $B_{h}^{x}$ approximates the differential operator $B^{x}$ with any prescribed order.

A function of a continuous [resp., discrete] argument that decays at infinity faster than any negative power of $|y|$ [resp., $|x|]$ is said to be rapidly decreasing. Let us define the Fourier transform of a grid function $f^{h}(x)$ by the formula

$$
\tilde{f}(\xi)=(2 \pi)^{-(n-1)} \sum_{x \in R_{h}^{n-1}} \exp \{-i(x, \xi)\} f^{h}(x) h^{n-1}, \quad \xi \in \mathbb{R}^{n-1} .
$$

This formula defines a $2 \pi h^{-1}$-periodic smooth function of the continuous argument $\xi$ whenever $f^{h}(x)$ is a rapidly decreasing grid function. In this last case, (3) is just a Fourier series expansion of the function $\tilde{f}(\xi)$ and the numbers $f^{h}(x)$ are the Fourier coefficients, given by the formula

$$
f^{h}(x)=\int_{\left|\xi_{1}\right| \leq \pi h^{-1}} \cdots \int_{\left|\xi_{n-1}\right| \leq \pi h^{-1}} \exp \{i(x, \xi)\} \tilde{f}(\xi) d \xi_{1} \cdots d \xi_{n-1}
$$

The inverse Fourier transform of a $2 \pi h^{-1}$-periodic function $\varphi(\xi)$ is defined to be the grid function $\tilde{\varphi}^{h}(x)$ given by the formula

$$
\tilde{\varphi}^{h}(x)=\int_{\left|\xi_{1}\right| \leq \pi h^{-1}} \cdots \int_{\left|\xi_{n-1}\right| \leq \pi h^{-1}} \exp \{i(x, \xi)\} \varphi(\xi) d \xi_{1} \cdots d \xi_{n-1} .
$$

Equations (4) and (5) establish a one-to-one correspondence between rapidly decreasing grid functions of a continuous argument. In particular, if $f^{h}(x)$ is a rapidly decreasing grid function, then

$$
\widehat{\tilde{f}}^{h}(x)=f^{h}(x)
$$

If $f^{h}(x)$ is a rapidly decreasing grid function, then the grid function $B_{h}^{x} f^{h}(x)$ exists and is given by (3) and we have the equality

$$
\widetilde{B^{x}}{ }_{h} f(\xi)=B(\xi h, h) \tilde{f}(\xi) .
$$


The function $B(\xi h, h)$ is obtained by replacing the operator $\Delta_{k \pm}$ in the right-hand side of equality (2) with the expression $\pm\left(\exp \left\{ \pm i \xi_{k} h\right\}-1\right)$, respectively, and is called the symbol of the difference operator. Since $\exp \left\{ \pm i \xi_{k} h\right\}$ is a bounded analytic $2 \pi h^{-1}$-periodic function, the symbol $B(\xi h, h)$ is a bounded analytic $2 \pi h^{-1}$-periodic function. It follows that for large $|\xi|$ one has the estimate

$$
|B(\xi h, h)| \leq M(h)|\xi|^{m}, \quad|\xi|^{2}=\left|\xi_{1}\right|^{2}+\cdots+\left|\xi_{n-1}\right|^{2} .
$$

Let us give the difference operator $A_{1 h}^{x}$ by the formula

$$
A_{1 h}^{x} u^{h}(x)=\sum_{2 m \leq|r| \leq S} a_{r}^{x} D_{h}^{r} u^{h}(x)+\delta u^{h}(x) .
$$

The coefficients are chosen in such a way that the operator $A_{1 h}^{x}$ approximates in a specified way the operator

$$
\sum_{|r|=2 m} a_{r}(x) \frac{\partial^{|r|}}{\partial x_{1}^{r_{1}} \cdots \partial x_{n-1}^{r_{n-1}}}+\delta
$$

We shall assume that for $\left|\xi_{k} h\right| \leq \pi$ and fixed $x$ the symbol $A_{1}^{x}(\xi h, h)$ of the operator $A_{1 h}^{x}-\delta$ satisfies the inequalities

$$
(-1)^{m} A_{1}^{x}(\xi h, h) \geq M_{1}|\xi|^{2 m}, \quad\left|\arg A_{1}^{x}(\xi h, h)\right| \leq \phi<\phi_{0} \leq \frac{\pi}{2} .
$$

In [12], Danelich considered the difference elliptic operator $A_{h}^{x}$ which is to an arbitrary high order of accuracy approximating the multi-dimensional elliptic operator $A^{x}$ defined by

$$
A^{x}=\sum_{|r|=2 m} a_{r}(x) \frac{\partial^{|r|}}{\partial x_{1}^{r_{1}} \cdots \partial x_{n-1}^{r_{n-1}}}+(-1)^{m} a(x) \frac{\partial^{2 m}}{\partial x_{n}^{2 m}}+\delta I
$$

with the domain

$$
D\left(A^{x}\right)=\left\{u \in C^{2 m}\left(\mathbb{R}_{n}^{+}\right):\left.u(x)\right|_{x_{n}=0}=\left.\frac{\partial u(x)}{\partial N}\right|_{x_{n}=0}=\cdots=\left.\frac{\partial^{m-1} u(x)}{\partial N^{m-1}}\right|_{x_{n}=0}=0\right\} .
$$

Here, $\delta>0$ is the sufficiently large number and $a(x)$ is a continuous function defined on $\mathbb{R}^{+}=\{x: x \geq 0\}$ with

$$
0<s_{1} \leq a(x) \leq s_{2}<\infty
$$

Let us define the grid space $\mathbb{R}_{h n}^{+}\left(0<h \leq h_{0}\right)$ as the set of all points of the space $\mathbb{R}_{n}^{+}$whose coordinates are given by

$$
x=\left(j_{1} h, \ldots, j_{n} h\right) \in \mathbb{R}_{n}^{+}, \quad j_{k} \in \mathbb{Z}, \quad k=\overline{1, n}, j_{n}=0,1,2, \ldots
$$


The number $h$ is called the step of the grid space. A function $\varphi^{h}(x)$ defined on $\mathbb{R}_{h n}^{+}$will be called a grid function. To the differential operator $A^{x}$ defined by (7), we assign the difference operator

$$
\begin{aligned}
& A_{h}^{x} \varphi^{h}(x)=A_{1 h}^{x} \varphi^{h}(x)+A_{2 h}^{x} \varphi^{h}(x)+\delta \varphi^{h}(x), \quad x \in \mathbb{R}_{h n}^{+}, \\
& A_{1 h}^{x} \varphi^{h}(x)=h^{-2 m} \sum_{2 m \leq|p| \leq S} a_{r}^{x} \Delta_{1-}^{p_{1}} \Delta_{1+}^{p_{2}} \cdots \Delta_{(n-1)-}^{p_{2 n-3}} \Delta_{(n-1)+}^{p_{2 n-2}} \varphi^{h}(x), \quad x \in \mathbb{R}_{h n}^{+}, \\
& A_{2 h}^{x} \varphi^{h}(x)=(-1)^{m} h^{-2 m} \sum_{\substack{2 m \leq r+s \leq S \\
s \leq m}} a_{r, s}^{x} \Delta_{n+}^{r} \Delta_{n-}^{s} \varphi^{h}(x), \quad x \in \mathbb{R}_{h n}^{+},
\end{aligned}
$$

which acts on functions defined on the entire space $\mathbb{R}_{h n}^{+}$. Here, $p \in \mathbb{R}_{2 n-2}^{+}$is a vector with nonnegative integers coordinates. The coefficients $a_{r}^{x}$ are chosen in such a way that the operator $A_{1 h}^{x}$ approximates in a specified way the operator

$$
\sum_{|r|=2 m} a_{r}(x) \frac{\partial^{|r|}}{\partial x_{1}^{r_{1}} \cdots \partial x_{n-1}^{r_{n-1}}} .
$$

We shall assume that for $\left|\xi_{k} h\right| \leq \pi$ and fixed $x$ the symbol $A_{1}^{x}(\xi h, h)$ of the operator $A_{1 h}^{x}$ satisfies the inequalities

$$
\begin{aligned}
& (-1)^{m} A_{1}^{x}\left(\xi^{\prime} h, h\right) \geq M_{1}\left|\xi^{\prime}\right|^{2 m}, \\
& \left|\arg A_{1}^{x}\left(\xi^{\prime} h, h\right)\right| \leq \phi<\phi_{0}<\pi, \quad \xi^{\prime}=\left(\xi_{1}, \ldots, \xi_{n-1}\right) .
\end{aligned}
$$

For the definition of the operator $A_{2 h}^{x}$, we will define $\varphi^{h}(x)$ which is extended to $\bar{\varphi}^{h}(x)$ defined on $\mathbb{R}_{h n}^{+}$and additionally on the points

$$
\left(x^{\prime}, x_{k}\right)=\left(x^{\prime}, k h\right) \notin \mathbb{R}_{h n}^{+}, \quad x^{\prime} \in \mathbb{R}_{h(n-1)}, k=-1,-2, \ldots,-(m-1),
$$

and

$$
\varphi^{h}\left(x^{\prime}, 0\right)=0, \quad h^{-k} \sum_{-k \leq s \leq s_{k}} \alpha_{s, k} \bar{\varphi}^{h}\left(x^{\prime}, s h\right)=0, \quad k=\overline{1, m-1}, \alpha_{-k, k} \neq 0 .
$$

The coefficients $\alpha_{s, k}$ are chosen in such a way that the expression

$$
h^{-k} \sum_{-k \leq s \leq s_{k}} \alpha_{s, k} \bar{\varphi}^{h}\left(x^{\prime}, s h\right)
$$

approximates in a specified way the expression $\varphi^{(k)}\left(x^{\prime}, 0\right)$. The coefficients $a_{r, s}^{x}$ are chosen in such a way that the operator $h^{-k} \sum_{-k \leq s \leq s_{k}} \alpha_{s, k} \bar{\varphi}^{h}\left(x^{\prime}, s h\right)$ approximates in a specified way the operator $A_{2}^{x}$. We shall assume that the operator $A_{2 h}^{x}$ approximates the differential operator $A_{2}^{x}$ with any prescribed order.

In the present paper, a Green's function is assigned. The organization of the present paper is as follows. In Section 3, the main theorem on the structure of fractional spaces $E_{\alpha}\left(\dot{C}_{h}\left(\mathbb{R}_{h n}^{+}\right), A_{h}^{x}\right)$ generated by $A_{h}^{x}$ is investigated. In Section 4 , applications on theorems on well-posedness in a Hölder space of parabolic and elliptic problems are presented. Finally, the conclusion is given. 


\section{Green's function}

Theorem 1 [12] Suppose that assumptions (8). Let the function a (x) satisfy the estimate

$$
|a(x)-a(y)| \leq s_{3}|x-y|^{\alpha} \quad\left(0<\alpha \leq 1 ; x, y \in \mathbb{R}_{h 1}^{+}\right) .
$$

Then, for sufficiently large positive $\delta$ and $\operatorname{Re} \lambda \geq 0$, there exists a unique solution of the resolvent equation

$$
A_{h}^{x} u^{h}(x)+\lambda u^{h}(x)=f^{h}(x), \quad x \in \mathbb{R}_{h n}^{+}
$$

and the following formula:

$$
f^{h}(y) h^{n}\left(A_{h}^{x}+\lambda I\right)^{-1} f^{h}(x)=\sum_{y \in \mathbb{R}_{h n}^{+}} G_{h}(x, y ; \lambda)
$$

holds. Here, $G_{h}(x, y ; \lambda)$ is the Green's function of the resolvent equation (13).

Lemma 1 The following identities hold:

$$
\begin{aligned}
& \sum_{y \in \mathbb{R}_{h n}^{+}} G_{h}(0, y ; \lambda) h^{n}=0, \\
& \sum_{y \in \mathbb{R}_{h n}^{+}} G_{h}(x, y ; \lambda) h^{n}=\frac{1}{\delta+\lambda}\left(1-v^{h}(x)\right), \quad x_{n}>h, x \in \mathbb{R}_{h n}^{+},
\end{aligned}
$$

where $v^{h}(x)$ is the solution of the following problem:

$$
\left\{\begin{array}{l}
h^{-2 m} \sum_{2 m \leq|p| \leq S} a_{r}^{x} \Delta_{1-}^{p_{1}} \Delta_{1+}^{p_{2}} \cdots \Delta_{(n-1)-}^{p_{2 n-3}} \Delta_{(n-1)+}^{p_{2 n-2}} v^{h}(x) \\
\quad+(-1)^{m} h^{-2 m} \sum_{2 m \leq r+s \leq S} a_{r, s}^{x} \Delta_{+}^{r} \Delta_{-}^{s} v^{h}(x)+(\delta+\lambda) v^{h}(x)=0, \quad x \in \mathbb{R}_{h n}^{+}, \\
v^{h}\left(x^{\prime}, 0\right)=1, \quad h^{-k} \sum_{-k \leq s \leq s_{k}} \alpha_{s, k} \bar{v}^{h}\left(x^{\prime}, s h\right)=0, \quad k=\overline{1, m-1}, \alpha_{-k, k} \neq 0, \\
x^{\prime} \in \mathbb{R}_{h(n-1) .}
\end{array}\right.
$$

Proof We consider the problem

$$
\left\{\begin{array}{l}
h^{-2 m} \sum_{2 m \leq|p| \leq S} a_{r}^{x} \Delta_{1-}^{p_{1}} \Delta_{1+}^{p_{2}} \cdots \Delta_{(n-1)-}^{p_{2 n-3}} \Delta_{(n-1)+}^{p_{2 n-2}} h^{h}(x) \\
\quad+(-1)^{m} h^{-2 m} \sum_{2 m \leq r+s \leq S} a_{r, s} \Delta_{+}^{r} \Delta_{-}^{s} u^{h}(x)+(\delta+\lambda) u^{h}(x)=f^{h}(x), \quad x \in \mathbb{R}_{h 1}^{+}, \\
u^{h}\left(x^{\prime}, 0\right)=1, \quad h^{-k} \sum_{-k \leq s \leq s_{k}} \alpha_{s, k} \bar{u}^{h}\left(x^{\prime}, s h\right)=0, \quad k=\overline{1, m-1}, \alpha_{-k, k} \neq 0, \\
x^{\prime} \in \mathbb{R}_{h(n-1) .}
\end{array}\right.
$$

We have

$$
u^{h}(x)=\sum_{y \in \mathbb{R}_{h n}^{+}} G_{h}(x, y ; \lambda) f^{h}(y) h^{n}+v^{h}(x) \frac{1}{\delta+\lambda}, \quad x \in \mathbb{R}_{h n}^{+} .
$$

Since for $f^{h}(x)=1, u^{h}(x)=\frac{1}{\delta+\lambda}$ is the solution of (13), we get

$$
\frac{1}{\delta+\lambda}=\sum_{y \in \mathbb{R}_{h n}^{+}} G_{h}(x, y ; \lambda) h^{n}+v^{h}(x) \frac{1}{\delta+\lambda}, \quad x \in \mathbb{R}_{h n}^{+} .
$$


Then

$$
\sum_{y \in \mathbb{R}_{h n}^{+}} G_{h}(x, y ; \lambda) h^{n}=\frac{1}{\delta+\lambda}\left(1-v^{h}(x)\right), \quad x \in \mathbb{R}_{h n}^{+} .
$$

Identity (16) is proved. Since $v^{h}(0)=1$, we have

$$
\frac{1}{\delta+\lambda}=\sum_{y \in \mathbb{R}_{h n}^{+}} G_{h}(0, y ; \lambda) h^{n}+\frac{1}{\delta+\lambda} .
$$

Thus follows (15). Lemma 1 is proved.

For any $x_{0} \in \mathbb{R}_{h n}^{+}$, we have

$$
G_{h}(x, y ; \lambda)=G_{h}^{x_{0}}(x, y ; \lambda)+\sum_{y \in \mathbb{R}_{h n}^{+}} G_{h}(x, z ; \lambda)\left[A_{h}^{x_{0}}-A_{h}^{z}\right] G_{h}^{x_{0}}(z, y ; \lambda) h^{n},
$$

where $G_{h}^{x_{0}}(x, y ; \lambda)$ is the Green's function of the operator $A_{h}^{x_{0}}$.

Moreover, applying (8), (10), (11), and (12) conditions, one proved the following pointwise estimates of the Green's function $G_{h}(x, y ; \lambda)$ of the resolvent equation and its difference derivatives:

$$
\left|G_{h}(x, y ; \lambda)\right| \leq M_{1} \exp \left\{-b|\lambda+\delta|^{p}|x-y|\right\}|\lambda+\delta|^{n p-1}
$$

for $2 m>n$,

$$
\left|G_{h}(x, y ; \lambda)\right| \leq M_{1} \exp \left\{-b|\lambda+\delta|^{p}|x-y|\right\}\left[1+\ln \left\{\left(|x-y||\lambda+\delta|^{p}\right)^{-1}+1\right\}\right]
$$

for $2 m=n$,

$$
\left|G_{h}(x, y ; \lambda)\right| \leq M_{1} \exp \left\{-b|\lambda+\delta|^{p}|x-y|\right\}|x-y|^{2 m-n}
$$

for $2 m<n$, and its derivatives respect to $x$

$$
\left|D_{h}^{r} G_{h}(x, y ; \lambda)\right| \leq M_{1} \exp \left\{-b|\lambda+\delta|^{p}|x-y|\right\}|\lambda+\delta|^{(n+r) p-1}
$$

for $2 m-r>n$,

$$
\left|D_{h}^{r} G_{h}(x, y ; \lambda)\right| \leq M_{1} \exp \left\{-b|\lambda+\delta|^{p}|x-y|\right\}\left[1+\ln \left\{\left(|x-y||\lambda+\delta|^{p}\right)^{-1}+1\right\}\right]
$$

for $2 m-r=n$,

$$
\left|D_{h}^{r} G_{h}(x, y ; \lambda)\right| \leq M_{1} \exp \left\{-b|\lambda+\delta|^{p}|x-y|\right\}|x-y|^{2 m-n-r}
$$

for $2 m-r<n$, where $p=\frac{1}{2 m}, a>0, M_{1}>0$ for $0<|\lambda| \leq L h^{-2 m}, \lambda \in Q_{\psi} \cup\{0\}$, and

$$
\left|G_{h}(x, y ; \lambda)\right| \leq M_{1} \exp \left\{-b h^{-1}|x-y|\right\} h^{2 m-n}\left(|\delta+\lambda| h^{2 m}+1\right)^{-1},
$$




$$
\left|D_{h}^{r} G_{h}(x, y ; \lambda)\right| \leq M_{1} \exp \left\{-b h^{-1}|x-y|\right\} h^{2 m-n-r}\left(|\delta+\lambda| h^{2 m}+1\right)^{-1}
$$

for $|\lambda|>L h^{-2 m}$. Here $p=\frac{1}{2 m}, b>0, M_{1}>0$.

Note that under assumptions (8) and (10) there exists a unique solution $v^{h}(x)$ of problem (17) and the following estimate holds:

$$
\left|v^{h}(x)\right| \leq M_{1} \exp \left\{-b|\lambda+\delta|^{p}|x|\right\}
$$

for $0<|\lambda| \leq L h^{-2 m}, \lambda \in Q_{\psi} \cup\{0\}$ and

$$
\left|v^{h}(x)\right| \leq M_{1} \exp \left\{-b h^{-1}|x|\right\}\left(|\delta+\lambda| h^{2 m}+1\right)^{-1},
$$

for $|\lambda|>L h^{-2 m}$.

Lemma $2[2]|\lambda|>L h^{-2 m}$, where $L>0$ large enough. Then

$$
\left|G_{h}(x, y ; \lambda)\right| \leq M_{1} \exp \left\{-b h^{-1}|x-y|\right\} h^{2 m}\left(|\delta+\lambda| h^{2 m}+1\right)^{-2}|x-y|^{-n}, \quad x \neq y .
$$

By (25) and (29), we obtain the estimate

$$
\begin{aligned}
& \left|G_{h}(x, y ; \lambda)\right| \\
& \quad \leq M_{1} \exp \left\{-b h^{-1}|x-y|\right\} h^{-1+2 m(1+\mu)} \\
& \quad \times\left(|\delta+\lambda| h^{2 m}+1\right)^{-(1+2 m \mu)}|x-y|^{-2 m \mu}, \quad x \neq y .
\end{aligned}
$$

Moreover, applying the Green's function of $A_{h}^{x}$ the following results were proved.

Theorem 2 [12] $A_{h}^{x}$ is a positive operator in the space $\dot{C}\left(\mathbb{R}_{h n}^{+}\right)$of all mesh functions $\varphi^{h}(x)$ defined on $\mathbb{R}_{\text {hn }}^{+}$with the norm

$$
\left\|\varphi^{h}\right\|_{\dot{C}_{h}}=\sup _{x \in \mathbb{R}_{h n}^{+}}\left|\varphi^{h}(x)\right|
$$

with the spectral angle $\varphi\left(A_{h}^{x}, \dot{C}_{h}\right)=\pi-\psi$.

Let $C_{h}^{\beta}=C^{\beta}\left(\mathbb{R}_{h n}^{+}\right)$be the Hölder space of all mesh functions $\varphi^{h}(x)$ defined on $\mathbb{R}_{h n}^{+}$satisfying a Hölder condition with the indicator $\beta \in(0,1)$ with the norm

$$
\left\|\varphi^{h}\right\|_{C_{h}^{\beta}}=\left\|\varphi^{h}\right\|_{C_{h}}+\sup _{\substack{x, y \in \mathbb{R}_{h n}^{+} \\ x \neq y}} \frac{\left|\varphi^{h}(x)-\varphi^{h}(y)\right|}{|x-y|^{\beta}} .
$$

One proved the strong positivity of $A_{h}^{x}$ in the Banach space $\dot{C}_{h}=\dot{C}\left(\mathbb{R}_{h n}^{+}\right)$(difference ana$\log$ of $\dot{C}\left(\mathbb{R}_{n}^{+}\right)$) for sufficiently large positive $\delta$. Passing to limit when $h \rightarrow 0$, we can get the strong positivity of differential operator $A^{x}$ in the Banach space $\dot{C}\left(\mathbb{R}_{n}^{+}\right)$. 


\section{The structure of fractional spaces $E_{\alpha}\left(\dot{C}_{h}\left(\mathbb{R}_{h n}^{+}\right), A_{h}^{x}\right)$}

Theorem 3 Suppose $2 m \alpha \in(0,1)$. Then the norms of the spaces $E_{\alpha}\left(\dot{C}_{h}, A_{h}^{x}\right)$ and $\dot{C}^{2 m \alpha}\left(\mathbb{R}_{h n}^{+}\right)$ are equivalent. Here,

$$
\begin{gathered}
\dot{C}_{h}^{2 m \alpha}=\left\{\varphi^{h}: \varphi^{h} \in C_{h}^{2 m \alpha}, \varphi^{h}\left(x^{\prime}, 0\right)=0, h^{-k} \sum_{-k \leq s \leq s_{k}} \alpha_{s, k} \bar{\varphi}^{h}\left(x^{\prime}, s h\right)=0,\right. \\
\left.k=\overline{1, m-1}, \alpha_{-k, k} \neq 0, x^{\prime} \in \mathbb{R}_{h(n-1)}\right\} .
\end{gathered}
$$

Proof Assume that $f^{h} \in \dot{C}^{2 m \alpha}\left(\mathbb{R}_{h n}^{+}\right)$. Let $x \in \mathbb{R}_{h n}^{+}$and $\lambda>0$ be fixed. Using equation (14) and identity (16), we can write

$$
\begin{aligned}
A_{h}^{x}\left(A_{h}^{x}+\lambda\right)^{-1} f^{h}(x) & \\
= & \frac{\delta}{\lambda+\delta} f^{h}(x)+\lambda \sum_{y \in \mathbb{R}_{n}^{+}} G_{h}(x, y ; \lambda)\left[f^{h}(x)-f^{h}(y)\right] h^{n} \\
& \quad+\frac{\lambda}{\lambda+\delta} v^{h}(x)\left(f^{h}(x)-f^{h}\left(x_{1}, \ldots, x_{n-1}, 0\right)\right)+\frac{\lambda}{\lambda+\delta} v^{h}(x) f^{h}\left(x_{1}, \ldots, x_{n-1}, 0\right) .
\end{aligned}
$$

Since $f^{h}\left(x_{1}, \ldots, x_{n-1}, 0\right)=0$, we have

$$
\begin{aligned}
A_{h}^{x}\left(A_{h}^{x}+\lambda\right)^{-1} f^{h}(x)= & \frac{\delta}{\lambda+\delta} f^{h}(x)+\lambda \sum_{y \in \mathbb{R}_{n}^{+}} G_{h}(x, y ; \lambda)\left[f^{h}(x)-f^{h}(y)\right] h^{n} \\
& +\frac{\lambda}{\lambda+\delta} v^{h}(x)\left(f^{h}(x)-f^{h}\left(x_{1}, \ldots, x_{n-1}, 0\right)\right) .
\end{aligned}
$$

Using equation (31) and the triangle inequality, and the definition of the $\dot{C}^{2 m \alpha}\left(\mathbb{R}_{h n}^{+}\right)$-norm, we have

$$
\begin{aligned}
& \left|\lambda^{\alpha} A_{h}^{x}\left(A_{h}^{x}+\lambda\right)^{-1} f^{h}(x)\right| \\
& \quad \leq\left[\frac{\lambda^{\alpha} \delta}{\lambda+\delta}+\lambda^{\alpha+1} \sum_{y \in \mathbb{R}_{n}^{+}}\left|G_{h}(x, y ; \lambda)\right||x-y|^{2 m \alpha} h^{n}+\frac{\lambda^{\alpha+1}}{\lambda+\delta}|v(x)||x|^{2 m \alpha}\right]\left\|f^{h}\right\|_{\dot{C}^{2 m \alpha}\left(\mathbb{R}_{h n}^{+}\right)} \\
& \quad=\left[I_{1}+I_{2}+I_{3}\right]\left\|f^{h}\right\|_{\dot{C}^{2 m \alpha}\left(\mathbb{R}_{h n}^{+}\right)} .
\end{aligned}
$$

We will estimate $I_{i}, i=1,2,3$, separately. First, let us estimate $I_{1}$. Clearly, using the estimate

$$
\frac{\lambda^{\alpha} \delta^{1-\alpha}}{\lambda+\delta} \leq 1
$$

we get

$$
I_{1} \leq \delta^{\alpha}
$$

for any $\lambda>0$. 
We will consider two cases: $\lambda>L h^{-2 m}$ and $\lambda \leq L h^{-2 m}$. First, let $\lambda>L h^{-2 m}$. Using estimate (30), we have

$$
\begin{aligned}
I_{2} & \leq M_{1} \lambda^{\alpha+1} \sum_{y \in \mathbb{R}_{n}^{+}} e^{-b h^{-1}|x-y|} h^{-n+2 m(1+\alpha)}\left((\lambda+\delta) h^{2 m}+1\right)^{-(1+2 m \alpha)} h^{n} \\
& \leq M_{2} \frac{\left(\lambda h^{2 m}\right)^{\alpha} \lambda}{\left((\lambda+\delta) h^{2 m}\right)^{2 m \alpha}(\lambda+\delta)} \leq M_{3}
\end{aligned}
$$

for any $\lambda>0$ and $x \in \mathbb{R}_{h n}^{+}$. From estimate (27) and the following inequality:

$$
(a t)^{\theta} e^{-a t} \leq M, \quad a>0, \theta \in[0,1]
$$

it follows that

$$
\begin{aligned}
I_{3} & \leq M_{1} \frac{\lambda^{\alpha+1}}{\lambda+\delta} e^{-a h^{-1}|x|}\left((\lambda+\delta) h^{2 m}+1\right)^{-1}|x|^{2 m \alpha} \\
& \leq M_{2} \frac{\lambda^{\alpha+1} h^{2 m \alpha}}{(\lambda+\delta)\left((\lambda+\delta) h^{2 m}+1\right)} \leq M_{3}
\end{aligned}
$$

for any $\lambda>0$ and $x \in \mathbb{R}_{h n}^{+}$. Combining estimates (32)-(35), we get

$$
\left|\lambda^{\alpha} A_{h}^{x}\left(A_{h}^{x}+\lambda\right)^{-1} f^{h}(x)\right| \leq M(\alpha)
$$

for any $\lambda>0$ and $x \in \mathbb{R}_{h n}^{+}$.

Second, let $\lambda \leq L h^{-2 m}$. We consider three cases: $2 m>n, 2 m=n$, and $2 m<n$.

In the first case, using estimate (19), applying inequality (34), and the definition of the $\dot{C}^{2 m \alpha}\left(\mathbb{R}_{h n}^{+}\right)$-norm, we have

$$
\begin{aligned}
I_{2} & \leq M_{1} \lambda^{\alpha+1} \sum_{y \in \mathbb{R}_{h n}^{+}} e^{-b(\lambda+\delta)^{\frac{1}{2 m}}|x-y|}(\lambda+\delta)^{\frac{n}{2 m}-1} \\
& \leq M_{2} \frac{\lambda^{\alpha+1}}{(\lambda+\delta)^{1-\frac{n}{2 m}+\alpha+\frac{\beta}{2 m}+\frac{n}{2 m}}} \leq M_{3}
\end{aligned}
$$

for any $\lambda>0$ and $x \in \mathbb{R}_{h n}^{+}$.

In the second case, applying estimate (20), and inequality (34), we have

$$
\begin{aligned}
I_{2} & \leq M_{1} \lambda^{\alpha+1} \sum_{y \in \mathbb{R}_{h n}^{+}} e^{-b(\lambda+\delta)^{\frac{1}{2 m}}|x-y|}\left[1+\ln \left\{\left(|x-y|(\lambda+\delta)^{\frac{1}{2 m}}\right)^{-1}+1\right\}\right]|x-y|^{2 m \alpha+\beta} h^{n} \\
& \leq M_{2} \frac{\lambda^{\alpha+1}}{(\lambda+\delta)^{\alpha+\frac{n}{2 m}+\frac{\beta}{2 m}}} \leq M_{3}
\end{aligned}
$$

for any $\lambda>0$ and $x \in \mathbb{R}_{h n}^{+}$.

In the third case, using estimate (21) we obtain

$$
\begin{aligned}
I_{2} & \leq M_{1} \lambda^{\alpha+1} \sum_{y \in \mathbb{R}_{h n}^{+}} e^{-b(\lambda+\delta)^{\frac{1}{2 m}}|x-y|}(\lambda+\delta)^{\frac{n}{2 m}-1}|x-y|^{2 m-n}|x-y|^{2 m \alpha+\beta} h^{n} \\
& \leq M_{2} \frac{\lambda^{\alpha+1}}{(\lambda+\delta)^{\frac{2 m-n+2 m \alpha+\beta}{2 m}-\frac{n}{2 m}}} \leq M_{3}
\end{aligned}
$$


for any $\lambda>0$ and $x \in \mathbb{R}_{h n}^{+}$. Estimates (37)-(39) result in

$$
I_{2} \leq M
$$

for any $\lambda>0$ and $x \in \mathbb{R}_{h n}^{+}$. From estimate (27) and inequality (34) it follows that

$$
I_{3} \leq M_{1} \frac{\lambda^{\alpha+1}}{\lambda+\delta} e^{-b(\lambda+\delta)^{\frac{1}{2 m}}|x|}|x|^{2 m \alpha+\beta} \leq M_{2}
$$

for any $\lambda>0$ and $x \in \mathbb{R}_{h n}^{+}$. Combining estimates (32), (40), and (41), we have

$$
\left|\lambda^{\alpha} A_{h}^{x}\left(A_{h}^{x}+\lambda\right)^{-1} f^{h}(x)\right| \leq M(\alpha)\left\|f^{h}\right\|_{\dot{C}^{2 m \alpha}}
$$

for any $\lambda>0$ and $x \in \mathbb{R}_{h n}^{+}$.

Combining estimates (36) and (42), we get

$$
\sup _{\lambda>0} \sup _{x \in \mathbb{R}_{h n}^{+}}\left|\lambda^{\alpha} A_{h}^{x}\left(A_{h}^{x}+\lambda\right)^{-1} f^{h}(x)\right| \leq M(\alpha)\left\|f^{h}\right\|_{\dot{C}^{2 m \alpha}} .
$$

Now, we will prove the opposite inequality. Using the definition of $E_{\alpha}\left(\dot{C}_{h}, A_{h}^{x}\right)$, we obtain

$$
\sup _{x \in \mathbb{R}_{n}^{+}}\left|f^{h}(x)\right| \leq\left\|f^{h}\right\|_{E_{\alpha}\left(\dot{C}_{h}, A_{h}^{x}\right)}
$$

for any $x \in \mathbb{R}_{h n}^{+}$. By Theorem 2, $A_{h}^{x}$ is a positive operator in the Banach space $E_{\alpha}\left(\dot{C}_{h}, A_{h}^{x}\right)$. Hence, for $f^{h} \in E_{\alpha}\left(\dot{C}_{h}, A_{h}^{x}\right)$, we have

$$
f^{h}=\int_{0}^{\infty} A_{h}^{x}\left(A_{h}^{x}+\lambda\right)^{-2} V d \lambda
$$

It follows from equation (14) and equation (45) that

$$
\begin{aligned}
f^{h}(x) & =\int_{0}^{\infty}\left(A_{h}^{x}+\lambda\right)^{-1} A_{h}^{x}\left(A_{h}^{x}+\lambda\right)^{-1} f^{h}(x) d \lambda \\
& =\int_{0}^{\infty} \sum_{y \in \mathbb{R}_{h n}^{+}} G_{h}(x, y ; \lambda) A_{h}^{x}\left(A_{h}^{x}+\lambda\right)^{-1} f^{h}(y) h^{n} d \lambda .
\end{aligned}
$$

Let $x, \tau \in \mathbb{R}_{h n}^{+}$be fixed. We will consider two cases: $\lambda>L h^{-2 m}$ and $\lambda \leq L h^{-2 m}$. First, let $\lambda>L h^{-2 m}$. Using equation (46) and Lemma 2 , we have

$$
\frac{f^{h}(x+\tau)-f^{h}(x)}{|\tau|^{2 m \alpha}}=\int_{0}^{\infty} \sum_{y \in \mathbb{R}_{h n}^{+}} \frac{\left[G_{h}(x+\tau, y ; \lambda)-G_{h}(x, y ; \lambda)\right]}{|\tau|^{2 m \alpha} \lambda^{\alpha}} A_{h}^{x}\left(A_{h}^{x}+\lambda\right)^{-1} f^{h}(y) h^{n} d \lambda .
$$


From the triangle inequality and the definition of the $E_{\alpha}\left(\dot{C}_{h}, A_{h}^{x}\right)$-norm it follows that

$$
\begin{aligned}
\left|\frac{f^{h}(x+\tau)-f^{h}(x)}{|\tau|^{2 m \alpha}}\right| \leq & {\left[\int_{0}^{\frac{1}{|\tau|^{2 m}}} \sum_{y \in \mathbb{R}_{h n}^{+}} \frac{\left|G_{h}(x+\tau, y ; \lambda)-G_{h}(x, y ; \lambda)\right|}{|\tau|^{2 m \alpha} \lambda^{\alpha}} h^{n} d \lambda\right.} \\
& \left.+\int_{\frac{1}{|\tau|^{2 m}}}^{\infty} \sum_{y \in \mathbb{R}_{h n}^{+}} \frac{\left|G_{h}(x+\tau, y ; \lambda)-G_{h}(x, y ; \lambda)\right|}{|\tau|^{2 m \alpha} \lambda^{\alpha}} h^{n} d \lambda\right]\left\|f^{h}\right\|_{E_{\alpha}\left(\dot{C}_{h}, A_{h}^{x}\right)} \\
= & {\left[L_{1}+L_{2}\right]\left\|f^{h}\right\|_{E_{\alpha}\left(\dot{C}_{h}, A_{h}^{x}\right)} . }
\end{aligned}
$$

We will estimate $L_{1}$ and $L_{2}$. Let us estimate $L_{1}$. From the Lagrange theorem and estimate (26), we have, for some $x^{*}$ between $x, x+\tau$,

$$
\begin{aligned}
L_{1} & \leq M_{1} \int_{0}^{\frac{1}{|\tau|^{2 m}}} \sum_{y \in \mathbb{R}_{h n}^{+}} \frac{|\tau| D_{h}^{1} G_{h}\left(x^{*}, y ; \lambda\right)}{|\tau|^{2 m \alpha} \lambda^{\alpha}} h^{n} d \lambda \\
& \leq M_{2} \int_{0}^{\frac{1}{|\tau|^{2 m}}} \sum_{y \in \mathbb{R}_{h n}^{+}} \frac{|\tau| e^{-b h^{-1}\left|x^{*}-y\right|} h^{2 m-1-n}\left((\lambda+\delta) h^{2 m}+1\right)^{-1}}{|\tau|^{2 m \alpha} \lambda^{\alpha}} h^{n} d \lambda \\
& \leq M_{3}(\alpha) \int_{0}^{\frac{1}{|\tau|^{2 m}}} \frac{|\tau|}{|\tau|^{2 m \alpha} \lambda^{\alpha+1-\frac{1}{2 m}}} d \lambda \leq M_{4}(\alpha)
\end{aligned}
$$

for any $\lambda>0$ and $x \in \mathbb{R}_{h n}^{+}$. Let us estimate $L_{2}$. The Lagrange theorem, estimate (25), and the inequality (34) yield

$$
\begin{aligned}
L_{2} \leq & M_{1}\left[\int_{\frac{1}{|\tau|^{2 m}}}^{\infty} \sum_{y \in \mathbb{R}_{h n}^{+}} \frac{e^{-b h^{-1}|x+\tau-y|} h^{2 m-n}\left((\lambda+\delta) h^{2 m}+1\right)^{-1}}{|\tau|^{2 m \alpha} \lambda^{\alpha}} h^{n} d \lambda\right. \\
& \left.+\int_{\frac{1}{|\tau|^{2 m}}}^{\infty} \sum_{y \in \mathbb{R}_{h n}^{+}} \frac{e^{-b h^{-1}|x-y|} h^{2 m-n}\left((\lambda+\delta) h^{2 m}+1\right)^{-1}}{|\tau|^{2 m \alpha} \lambda^{\alpha}} h^{n} d \lambda\right] \\
\leq & M_{2} \int_{\frac{1}{|\tau|^{2 m}}}^{\infty} \frac{1}{|\tau|^{2 m \alpha} \lambda^{\alpha+1}} d \lambda \leq M_{3}(\alpha)
\end{aligned}
$$

for any $\lambda>0$ and $x \in \mathbb{R}_{h n}^{+}$. Then, combining estimates (47) and (48), we get

$$
\left|\frac{f^{h}(x+\tau)-f^{h}(x)}{|\tau|^{2 m \alpha}}\right| \leq M\left\|f^{h}\right\|_{E_{\alpha}} .
$$

Second, let $\lambda \leq L h^{-2 m}$. We will estimate $L_{1}$ and $L_{2}$. Let us estimate $L_{1}$. We consider three cases: $2 m>n, 2 m=n$, and $2 m<n$. When $2 m>n$ we consider two separate cases: $2 m-1=$ $n$ and $2 m-1>n$. First, let $2 m-1>n$. From the Lagrange theorem, estimate (22), the inequality (34) we have, for some $x^{*}$ between $x, x+\tau$,

$$
\begin{aligned}
L_{1} & \leq M_{1} \int_{0}^{\frac{1}{|\tau|^{2 m}}} \sum_{y \in \mathbb{R}_{h n}^{+}} \frac{|\tau| e^{-a(\lambda+\delta)^{\frac{1}{2 m}}}\left|x^{*}-y\right|}{|\tau|^{2 m \alpha} \lambda^{\alpha}(\lambda+\delta)^{1-\frac{n+1}{2 m}}} h^{n} d \lambda \\
& \leq M_{2} \int_{0}^{\frac{1}{|\tau|^{2 m}}} \frac{|\tau|}{|\tau|^{2 m \alpha} \lambda^{\alpha+1-\frac{1}{2 m}}} d \lambda \leq M_{3}(\alpha)
\end{aligned}
$$


for any $\lambda>0$ and $x \in \mathbb{R}_{h n}^{+}$. Second, let $2 m-1=n$. From the Lagrange theorem, estimate (23), and the inequality (34), we have, for some $x^{*}$ between $x, x+\tau$,

$$
\begin{aligned}
L_{1} & \leq M_{1} \int_{0}^{\frac{1}{|\tau|^{2 m}}} \sum_{y \in \mathbb{R}_{h n}^{+}} \frac{|\tau|}{|\tau|^{2 m \alpha} \lambda^{\alpha}} e^{-b(\lambda+\delta) \frac{1}{2 m}\left|x^{*}-y\right|}\left[1+\ln \left\{\left(\left|x^{*}-y\right|(\lambda+\delta)^{\frac{1}{2 m}}\right)^{-1}+1\right\}\right] h^{n} d \lambda \\
& \leq M_{2} \int_{0}^{\frac{1}{|\tau|^{2 m}}} \frac{|\tau|}{|\tau|^{2 m \alpha} \lambda^{\alpha+1-\frac{n}{2 m}}} d \lambda \leq M_{3}(\alpha)
\end{aligned}
$$

for any $\lambda>0$ and $x \in \mathbb{R}_{h n}^{+}$. In the cases $2 m=n$ and $2 m<n$, we have a situation where $2 m-1<n$. Using the Lagrange theorem, estimate (24), we have, for some $x^{*}$ between $x$, $x+\tau$,

$$
\begin{aligned}
L_{1} & \leq M_{1} \int_{0}^{\frac{1}{|\tau|^{2 m}}} \frac{|\tau|}{|\tau|^{2 m \alpha} \lambda^{\alpha}} \sum_{y \in \mathbb{R}_{h n}^{+}} e^{-a(\lambda+\delta)^{\frac{1}{2 m}}\left|x^{*}-y\right|}\left|x^{*}-y\right|^{2 m-n-1} h^{n} d \lambda \\
& \leq M_{2} \int_{0}^{\frac{1}{|\tau|^{2 m}}} \frac{|\tau|}{|\tau|^{2 m \alpha} \lambda^{\alpha+1-\frac{1}{2 m}}} d \lambda \leq M_{3}(\alpha)
\end{aligned}
$$

for any $\lambda>0$ and $x \in \mathbb{R}_{h n}^{+}$. Then, combining estimates (50)-(52), we get

$$
L_{1} \leq M(\alpha)
$$

for any $\lambda>0$ and $x \in \mathbb{R}_{h n}^{+}$. Let us estimate $L_{2}$. We consider three cases: $2 m>n, 2 m=n$, and $2 m<n$. First, let $2 m>n$. Using the triangle inequality, estimate (19), we get

$$
\begin{aligned}
L_{2} \leq & M_{1}\left[\int_{\frac{1}{|\tau|^{2 m}}}^{\infty} \sum_{y \in \mathbb{R}_{h n}^{+}} \frac{e^{-b(\lambda+\delta)^{\frac{1}{2 m}}|x+\tau-y|}}{|\tau|^{2 m \alpha} \lambda^{\alpha}(\lambda+\delta)^{1-\frac{n}{2 m}}} h^{n} d \lambda\right. \\
& \left.+\int_{\frac{1}{|\tau|^{2 m}}}^{\infty} \sum_{y \in \mathbb{R}_{h n}^{+}} \frac{e^{-b(\lambda+\delta)^{\frac{1}{2 m}}|x-y|}}{|\tau|^{2 m \alpha} \lambda^{\alpha}(\lambda+\delta)^{1-\frac{n}{2 m}}} h^{n} d \lambda\right] \\
\leq & M_{2} \int_{\frac{1}{|\tau|^{2 m}}}^{\infty} \frac{1}{|\tau|^{2 m \alpha} \lambda^{\alpha+1}} d \lambda \leq M_{3}(\alpha)
\end{aligned}
$$

for any $\lambda>0$ and $x \in \mathbb{R}_{h n}^{+}$. Second, let $2 m=n$. From the triangle inequality, estimate (20) it follows that

$$
\begin{aligned}
L_{2} \leq & M_{1}\left[\int_{\frac{1}{|\tau|^{2 m}}}^{\infty} \sum_{y \in \mathbb{R}_{h n}^{+}} \frac{1}{|\tau|^{2 m \alpha} \lambda^{\alpha}} e^{-b(\lambda+\delta)^{\frac{1}{2 m}}|x+\tau-y|}\right. \\
& \times\left[1+\ln \left\{\left(|x+\tau-y|(\lambda+\delta)^{\frac{1}{2 m}}\right)^{-1}+1\right\}\right] h^{n} d \lambda \\
& \left.+\int_{\frac{1}{|\tau|^{2 m}}}^{\infty} \sum_{y \in \mathbb{R}_{h n}^{+}} \frac{1}{|\tau|^{2 m \alpha} \lambda^{\alpha}} e^{-b(\lambda+\delta)^{\frac{1}{2 m}}|x-y|}\left[1+\ln \left\{\left(|x-y|(\lambda+\delta)^{\frac{1}{2 m}}\right)^{-1}+1\right\}\right] h^{n} d \lambda\right] \\
\leq & M_{2} \int_{\frac{1}{|\tau|^{2 m}}}^{\infty} \frac{1}{|\tau|^{2 m \alpha} \lambda^{\alpha+1}} d \lambda \leq M_{3}(\alpha)
\end{aligned}
$$


for any $\lambda>0$ and $x \in \mathbb{R}_{h n}^{+}$. Third, let $2 m<n$. Using the triangle inequality, estimate (21), we have

$$
\begin{aligned}
L_{2} \leq & M_{1}\left[\int_{\frac{1}{|\tau|^{2 m}}}^{\infty} \sum_{y \in \mathbb{R}_{h n}^{+}} \frac{e^{-b(\lambda+\delta)^{\frac{1}{2 m}}|x+\tau-y|}}{|\tau|^{2 m \alpha} \lambda^{\alpha}}|x+\tau-y|^{2 m-n} h^{n} d \lambda\right. \\
& \left.+\int_{\frac{1}{|\tau|^{2 m}}}^{\infty} \sum_{y \in \mathbb{R}_{h n}^{+}} \frac{e^{-b(\lambda+\delta)^{\frac{1}{2 m}}|x-y|}}{|\tau|^{2 m \alpha} \lambda^{\alpha}}|x-y|^{2 m-n} h^{n} d \lambda\right] \\
\leq & M_{2} \int_{\frac{1}{|\tau|^{2 m}}}^{\infty} \frac{1}{|\tau|^{2 m \alpha} \lambda^{\alpha+1}} d \lambda \leq M_{3}(\alpha) .
\end{aligned}
$$

Then, combining estimates (54)-(56), we have

$$
L_{2} \leq M(\alpha) .
$$

Estimates (53) and (57) yield

$$
\left|\frac{f^{h}(x+\tau)-f^{h}(x)}{|\tau|^{2 m \alpha}}\right| \leq M(\alpha)\left\|f^{h}\right\|_{E_{\alpha}} .
$$

Combining estimates (49) and (58), we obtain

$$
\sup _{\substack{x \in \mathbb{R}_{h n}^{+} \\ \tau \neq(0, \ldots, 0)}}\left|\frac{f^{h}(x+\tau)-f^{h}(x)}{|\tau|^{2 m \alpha}}\right| \leq M(\alpha)\left\|f^{h}\right\|_{E_{\alpha}} .
$$

From estimates (43) and (59) it follows that

$$
E_{\alpha}\left(\dot{C}_{h}, A_{h}^{x}\right) \subset \dot{C}^{2 m \alpha}\left(\mathbb{R}_{h n}^{+}\right) .
$$

This is the end of the proof of Theorem 3 .

From Theorem 2 and Theorem 3 follows the following result.

Theorem $4 A_{h}^{x}$ is a positive operator in the space $\dot{C}^{\beta}\left(\mathbb{R}_{h n}^{+}\right), \beta \in(0,1)$.

\section{Applications}

Now, we present some applications of Theorems 2-3.

First, we consider the difference schemes for the approximate solution of problem

$$
\left\{\begin{array}{l}
-\frac{\partial^{2} u(t, x)}{\partial t^{2}}+\sum_{|r|=2 m} a_{r}(x) \frac{\partial^{|r|} u(t, x)}{\partial x_{1}^{r_{1} \ldots \partial x_{n-1}^{n-1}}} \\
\quad+(-1)^{m} a(x) \frac{\partial^{2 m} u(t, x)}{\partial x^{2 m}}+\delta u(t, x)=f(t, x), \quad 0<t<T, x \in \mathbb{R}_{n}^{+}, \\
u(0, x)=\varphi(x), \quad u(T, x)=\psi(x), \quad x \in \mathbb{R}_{n}^{+}, \\
\left.u(t, x)\right|_{x_{n}=0}=\left.\frac{\partial u(t, x)}{\partial N}\right|_{x_{n}=0}=\cdots=\left.\frac{\partial^{m-1} u(t, x)}{\partial N^{m-1}}\right|_{x_{n}=0}=0, \quad x \in \mathbb{R}_{n}^{+}, 0 \leq t \leq T .
\end{array}\right.
$$

Here, $a_{r}(x), a(x), \varphi(x), \psi(x)$, and $f(t, x)$ are sufficiently smooth functions and they satisfy all compatibility conditions which guarantee that problem (60) has a smooth solution $u(t, x)$. 
Assume that uniform ellipticity holds. Note that problem (60) can be written in the form of the abstract boundary value problem

$$
\left\{\begin{array}{l}
-u^{\prime \prime}(t)+A u(t)=f(t), \quad 0<t<T \\
u(0)=\varphi, \quad u(T)=\psi
\end{array}\right.
$$

in a Banach space $E=\dot{C}_{h}$ with positive operator $A=A_{h}^{x}$ defined by (9) and (11). The discretization of problem (60) is carried out in two steps. In the first step, let us give the difference operator $A_{h}^{x}$ by equation (9). With the help of $A_{h}^{x}$ we arrive at the boundary value problem

$$
\left\{\begin{array}{l}
-\frac{d^{2} u^{h}(t, x)}{d t^{2}}+A_{h}^{x} u^{h}(t, x)=f^{h}(t, x), \quad 0<t<T, x \in \mathbb{R}_{h n}^{+}, \\
u^{h}(0, x)=\varphi^{h}(x), \quad u^{h}(T, x)=\psi^{h}(x), \quad x \in \mathbb{R}_{h n}^{+},
\end{array}\right.
$$

for an infinite system of ordinary differential equations.

In the second step, we replace problem (62) by the difference scheme

$$
\left\{\begin{array}{l}
-\frac{1}{\tau^{2}}\left(u_{k+1}^{h}(x)-2 u_{k}^{h}(x)+u_{k-1}^{h}(x)\right)+A_{h}^{x} u_{k}^{h}(x)=f_{k}^{h}(x), \\
f_{k}^{h}(x)=f^{h}\left(t_{k}, x\right), \quad t_{k}=k \tau, 1 \leq k \leq N-1, N \tau=T, x \in \mathbb{R}_{h n}^{+}, \\
u_{0}^{h}(x)=\varphi^{h}(x), \quad u_{N}^{h}(x)=\psi^{h}(x), \quad x \in \mathbb{R}_{h n}^{+},
\end{array}\right.
$$

for the approximate solution of boundary value problem (60).

Theorem 5 Let $0 \leq \mu<1$. Then the solution of difference scheme (63) satisfies the following stability estimate:

$$
\max _{0 \leq k \leq N}\left\|u_{k}^{h}\right\|_{\dot{C}_{h}^{\mu}} \leq M(\mu)\left[\left\|\varphi^{h}\right\|_{\dot{C}_{h}^{\mu}}+\left\|\varphi^{h}\right\|_{\dot{C}_{h}^{\mu}}+\max _{1 \leq k \leq N-1}\left\|f_{k}^{h}\right\|_{\dot{C}_{h}^{\mu}}\right]
$$

The proof of Theorem 5 is based on Theorem 2 and Theorem 4, on the positivity of the difference operator $A_{h}^{x}$ in the Banach space $\dot{C}_{h}^{\mu}$ for $0 \leq \mu<1$, and on the following abstract theorem on the stability of the difference scheme

$$
\left\{\begin{array}{l}
-\frac{1}{\tau^{2}}\left(u_{k+1}-2 u_{k}+u_{k-1}\right)+A u_{k}=f_{k}, \\
f_{k}=f\left(t_{k}\right), \quad t_{k}=k \tau, 1 \leq k \leq N-1, N \tau=T, u_{0}=\varphi, u_{N}=\psi
\end{array}\right.
$$

for the approximate solution of the abstract boundary value problem (61).

Theorem 6 [3] Let A be a positive operator in a Banach space E. Then, for the solution of difference scheme (64), the following stability inequality holds:

$$
\max _{0 \leq k \leq N}\left\|u_{k}\right\|_{E_{\alpha}} \leq M(\alpha)\left[\|\varphi\|_{E_{\alpha}}+\|\psi\|_{E_{\alpha}}+\max _{1 \leq k \leq N-1}\left\|f_{k}\right\|_{E_{\alpha}}\right]
$$


Theorem 7 The solution of difference scheme (63) satisfies the following almost coercive stability estimate:

$$
\begin{aligned}
\max _{1 \leq k \leq N-1} \| & \left\|\frac{1}{\tau^{2}}\left(u_{k+1}^{h}-2 u_{k}^{h}+u_{k-1}^{h}\right)\right\|_{\dot{C}_{h}} \\
& +h^{-2 m} \sum_{2 m \leq|p| \leq S}\left\|\Delta_{1-}^{p_{1}} \Delta_{1+}^{p_{2}} \cdots \Delta_{(n-1)-}^{p_{2 n-3}} \Delta_{(n-1)+}^{p_{2 n-2}} u^{h}\right\|_{\dot{C}_{h}}+h^{-2 m} \sum_{2 m \leq r+s \leq S}\left\|\Delta_{+}^{r} \Delta_{-}^{s} u^{h}\right\|_{\dot{C}_{h}} \\
\leq & M \ln \frac{1}{h}\left[h^{-2 m} \sum_{2 m \leq|p| \leq S}\left\|\Delta_{1-}^{p_{1}} \Delta_{1+}^{p_{2}} \cdots \Delta_{(n-1)-}^{p_{2 n-3}} \Delta_{(n-1)+}^{p_{2 n-2}} \varphi^{h}\right\|_{\dot{C}_{h}}\right. \\
& +h^{-2 m} \sum_{\substack{2 m \leq r+s \leq S \\
s \leq m}}\left\|\Delta_{+}^{r} \Delta_{-}^{s} \varphi^{h}\right\|_{\dot{C}_{h}}+h^{-2 m} \sum_{2 m \leq|p| \leq S}\left\|\Delta_{1-}^{p_{1}} \Delta_{1+}^{p_{2}} \cdots \Delta_{(n-1)-}^{p_{2 n-3}} \Delta_{(n-1)+}^{p_{2 n-2}} \psi^{h}\right\|_{\dot{C}_{h}} \\
& \left.+h^{-2 m} \sum_{\substack { 2 m \leq r+s \leq S \\
\begin{subarray}{c}{s \leq m \\
S \leq S{ 2 m \leq r + s \leq S \\
\begin{subarray} { c } { s \leq m \\
S \leq S } }\end{subarray}}\left\|\Delta_{+}^{r} \Delta_{-}^{s} \psi^{h}\right\|_{\dot{C}_{h}}\right]+M \ln \frac{1}{\tau+h} \max _{1 \leq k \leq N-1}\left\|f_{k}^{h}\right\|_{\dot{C}_{h}} .
\end{aligned}
$$

The proof of Theorem 7 is based on Theorem 2 on the positivity of an elliptic difference operator $A_{h}^{x}$ in the Banach space $\dot{C}_{h}$ and on the estimate

$$
\min \left\{\ln \frac{1}{\tau}, 1+\left|\ln \left\|A_{h}^{x}\right\|_{\dot{C}_{h} \rightarrow \dot{C}_{h}}\right|\right\} \leq M \ln \frac{1}{\tau+h}
$$

and on the following theorems on the almost coercive stability of difference scheme (64) and on the almost coercive stability of the elliptic difference problem.

Theorem 8 [3] Let $A$ be a positive operator in a Banach space E. Then, for the solution of difference scheme (64), the following almost coercive stability inequality holds:

$$
\begin{aligned}
& \max _{1 \leq k \leq N-1}\left\|\frac{1}{\tau^{2}}\left(u_{k+1}-2 u_{k}+u_{k-1}\right)\right\|_{E}+\max _{0 \leq k \leq N}\left\|A u_{k}\right\|_{E} \\
& \quad \leq M\left[\|A \varphi\|_{E}+\|A \psi\|_{E}+\min \left\{\ln \frac{1}{\tau}, 1+\left|\ln \|A\|_{E \rightarrow E}\right|\right\} \max _{1 \leq k \leq N-1}\left\|f_{k}\right\|_{E}\right] .
\end{aligned}
$$

Theorem 9 Under assumptions (8) and (10), the solution of the difference elliptic problem

$$
A_{h}^{x} u^{h}(x)=f^{h}(x), \quad x \in \mathbb{R}_{h n}^{+},
$$

satisfies the following almost coercive inequality:

$$
\begin{aligned}
& h^{-2 m} \sum_{2 m \leq|p| \leq S}\left\|\Delta_{1-}^{p_{1}} \Delta_{1+}^{p_{2}} \cdots \Delta_{(n-1)-}^{p_{2 n-3}} \Delta_{(n-1)+}^{p_{2 n-2}} u^{h}\right\|_{\dot{C}_{h}}+h^{-2 m} \sum_{\substack{2 m \leq r+s \leq S \\
s \leq m}}\left\|\Delta_{+}^{r} \Delta_{-}^{s} u^{h}\right\|_{\dot{C}_{h}} \\
& \quad \leq M \ln \frac{1}{h}\left\|f^{h}\right\|_{\dot{C}_{h}} .
\end{aligned}
$$

The proof of Theorem 9 uses the techniques introduced in [2] and it is based on estimates for the Green's function of the operator $A_{h}^{x}$ defined by (7). 
Theorem 10 Let $0<2 m \alpha<1$. Then, for the solution of difference scheme (63), the following coercive stability estimate holds:

$$
\begin{aligned}
& \max _{1 \leq k \leq N-1}\left\|\frac{1}{\tau^{2}}\left(u_{k+1}^{h}-2 u_{k}^{h}+u_{k-1}^{h}\right)\right\|_{\dot{C}_{h}^{2 m \alpha}} \\
& +h^{-2 m} \sum_{2 m \leq|p| \leq S}\left\|\Delta_{1-}^{p_{1}} \Delta_{1+}^{p_{2}} \cdots \Delta_{(n-1)-}^{p_{2 n-3}} \Delta_{(n-1)+}^{p_{2 n-2}} u^{h}\right\|_{\dot{C}_{h}^{2 m \alpha}} \\
& +h^{-2 m} \sum_{\substack{2 m \leq r+s \leq S \\
s \leq m}}\left\|\Delta_{+}^{r} \Delta_{-}^{s} u^{h}\right\|_{\dot{C}_{h}^{2 m \alpha}} \\
& \leq M(\alpha)\left[h^{-2 m} \sum_{2 m \leq|p| \leq S}\left\|\Delta_{1-}^{p_{1}} \Delta_{1+}^{p_{2}} \cdots \Delta_{(n-1)-}^{p_{2 n-3}} \Delta_{(n-1)+}^{p_{2 n-2}} \varphi^{h}\right\|_{\dot{C}_{h}^{2 m \alpha}}\right. \\
& +h^{-2 m} \sum_{\substack{2 m \leq r+s \leq S \\
s \leq m}}\left\|\Delta_{+}^{r} \Delta_{-}^{s} \varphi^{h}\right\|_{\dot{C}_{h}^{2 m \alpha}} \\
& +h^{-2 m} \sum_{2 m \leq|p| \leq S}\left\|\Delta_{1-}^{p_{1}} \Delta_{1+}^{p_{2}} \cdots \Delta_{(n-1)-}^{p_{2 n-3}} \Delta_{(n-1)+}^{p_{2 n-2}} \psi^{h}\right\|_{\dot{C}_{h}^{2 m \alpha}} \\
& \left.+h^{-2 m} \sum_{\substack{m \leq r+s \leq S \\
s \leq m}}\left\|\Delta_{+}^{r} \Delta_{-}^{s} \psi^{h}\right\|_{\dot{C}_{h}^{2 m \alpha}}+M(\alpha) \max _{1 \leq k \leq N-1}\left\|f_{k}^{h}\right\|_{\dot{C}_{h}^{2 m \alpha}}\right] .
\end{aligned}
$$

The proof of Theorem 10 is based on Theorem 3 on the structure of the fractional spaces $E_{\alpha}\left(\dot{C}_{h}, A_{h}^{x}\right)$, on Theorem 2 on the positivity of an elliptic difference operator $A_{h}^{x}$ in the Banach space $\dot{C}_{h}$, and on the following theorems on the structure of the fractional space $E_{\alpha}^{\prime}=E_{\alpha}\left(E, A^{1 / 2}\right)$, on the coercive stability of difference scheme (64), and on the coercive stability of the elliptic difference problem.

Theorem 11 [16] The spaces $E_{\alpha}(E, A)$ and $E_{2 \alpha}^{\prime}\left(A^{1 / 2}, E\right)$ coincide for any $0<\alpha<\frac{1}{2}$, and their norms are equivalent.

Theorem 12 [3] Let A be a strongly positive operator in a Banach space E. Then, for the solution of difference scheme (64), the following coercive stability inequality:

$$
\begin{gathered}
\max _{1 \leq k \leq N-1}\left\|\frac{1}{\tau^{2}}\left(u_{k+1}-2 u_{k}+u_{k-1}\right)\right\|_{E_{\alpha}}+\max _{0 \leq k \leq N}\left\|A u_{k}\right\|_{E_{\alpha}} \\
\leq M(\alpha)\left[\|A \varphi\|_{E_{\alpha}}+\|A \psi\|_{E_{\alpha}}+\max _{1 \leq k \leq N-1}\left\|f_{k}\right\|_{E_{\alpha}}\right]
\end{gathered}
$$

is valid.

Theorem 13 Let $0<\mu<1$. Then, under assumptions (8) and (10) for the solution of difference elliptic problem (66), we have the following coercive inequality:

$$
\begin{aligned}
& h^{-2 m} \sum_{2 m \leq|p| \leq S}\left\|\Delta_{1-}^{p_{1}} \Delta_{1+}^{p_{2}} \cdots \Delta_{(n-1)-}^{p_{2 n-3}} \Delta_{(n-1)+}^{p_{2 n-2}} u^{h}\right\|_{\dot{C}_{h}^{\mu}} \\
& +h^{-2 m} \sum_{2 m \leq r+S \leq S}\left\|\Delta_{+}^{r} \Delta_{-}^{s} u^{h}\right\|_{\dot{C}_{h}^{\mu}} \leq M(\mu)\left\|f^{h}\right\|_{\dot{C}_{h}^{\mu}} .
\end{aligned}
$$


The proof of Theorem 13 uses the techniques introduced in [3] and it is based on estimates for the Green's function of operator $A_{h}^{x}$ defined by (7).

Second, we consider the difference schemes for the approximate solution of problem

$$
\left\{\begin{array}{l}
-\frac{\partial^{2} u(t, x)}{\partial t^{2}}+\sum_{|r|=2 m} a_{r}(x) \frac{\partial^{|r|} u(t, x)}{\partial x_{1}^{11} \cdots \partial x_{n-1}^{x_{n-1}}} \\
\quad+(-1)^{m} a(x) \frac{\partial^{2 m} u(t, x)}{\partial x^{2 m}}+\delta u(t, x)=f(t, x), \quad 0<t<T, x \in \mathbb{R}_{n}^{+}, \\
u(0, x)=u(T, x), \quad u_{t}(0, x)=u_{t}(T, x), \quad x \in \mathbb{R}_{n}^{+}, \\
\left.u(t, x)\right|_{x_{n}=0}=\left.\frac{\partial u(t, x)}{\partial N}\right|_{x_{n}=0}=\cdots=\left.\frac{\partial^{m-1} u(t, x)}{\partial N^{m-1}}\right|_{x_{n}=0}=0, \quad x \in \mathbb{R}_{n}^{+}, 0 \leq t \leq T .
\end{array}\right.
$$

Here, $a_{r}(x), a(x)$, and $f(t, x)$ are sufficiently smooth functions and they satisfy every compatibility conditions which guarantee that problem (67) has a smooth solution $u(t, x)$. Assume that the assumption of the uniform ellipticity holds. The discretization of problem (67) is carried out in two steps. In the first step, let us give the difference operator $A_{h}^{x}$ by equation (9). With the help of $A_{h}^{x}$ we arrive at the boundary value problem

$$
\left\{\begin{array}{l}
-\frac{d^{2} u^{h}(t, x)}{d t^{2}}+A_{h}^{x} u^{h}(t, x)=f^{h}(t, x), \quad 0<t<T, x \in \mathbb{R}_{h n}^{+}, \\
u^{h}(0, x)=u^{h}(T, x), \quad u_{t}^{h}(0, x)=u_{t}^{h}(T, x), \quad x \in \mathbb{R}_{h n}^{+},
\end{array}\right.
$$

for an infinite system of ordinary differential equations.

In the second step, we replace problem (68) by the first order of approximation in the $t$ difference scheme

$$
\left\{\begin{array}{l}
-\frac{1}{\tau^{2}}\left(u_{k+1}^{h}(x)-2 u_{k}^{h}(x)+u_{k-1}^{h}(x)\right)+A_{h}^{x} u_{k}^{h}(x)=f_{k}^{h}(x), \\
f_{k}^{h}(x)=f^{h}\left(t_{k}, x\right), \quad t_{k}=k \tau, 1 \leq k \leq N-1, N \tau=T, x \in \mathbb{R}_{h n^{+},}, \\
u_{0}^{h}(x)=u_{N}^{h}(x), \quad u_{1}^{h}(x)-u_{0}^{h}(x)=u_{N}^{h}(x)-u_{N-1}^{h}(x), \quad x \in \mathbb{R}_{h n}^{+},
\end{array}\right.
$$

and the second order of approximation in the $t$ difference scheme

$$
\left\{\begin{array}{l}
-\frac{1}{\tau^{2}}\left(u_{k+1}^{h}(x)-2 u_{k}^{h}(x)+u_{k-1}^{h}(x)\right)+A_{h}^{x} u_{k}^{h}(x)=f_{k}^{h}(x), \\
f_{k}^{h}(x)=f^{h}\left(t_{k}, x\right), \quad t_{k}=k \tau, 1 \leq k \leq N-1, N \tau=T, x \in \mathbb{R}_{h n}^{+}, u_{0}^{h}(x)=u_{N}^{h}(x), \\
-u_{2}^{h}(x)+4 u_{1}^{h}(x)-3 u_{0}^{h}(x)=u_{N-2}^{h}(x)-4 u_{N-1}^{h}(x)+3 u_{N}^{h}(x), \quad x \in \mathbb{R}_{h n}^{+},
\end{array}\right.
$$

for the approximate solution of boundary value problem (67).

Theorem 14 Let $0 \leq \mu<1$. Then the solution of the difference schemes (69) and (70) satisfies the following stability estimate:

$$
\max _{0 \leq k \leq N}\left\|u_{k}^{h}\right\|_{\dot{C}_{h}^{\mu}} \leq M(\mu)\left[\left\|\varphi^{h}\right\|_{\dot{C}_{h}^{\mu}}+\left\|\varphi^{h}\right\|_{\dot{C}_{h}^{\mu}}+\max _{1 \leq k \leq N-1}\left\|f_{k}^{h}\right\|_{\dot{C}_{h}^{\mu}}\right]
$$

The proof of Theorem 14 is based on Theorems 2 and 4, on the positivity of the difference operator $A_{h}^{x}$ in the Banach space $\dot{C}_{h}^{\mu}, \mu \in[0,1)$, and on the following abstract theorem, and on the stability of the difference schemes (69) and (70). 
Theorem 15 [3] Let A be a positive operator in a Banach space E. Then, for the solution of difference schemes (69) and (70), the following stability inequality holds:

$$
\max _{0 \leq k \leq N}\left\|u_{k}\right\|_{E_{\alpha}} \leq M(\alpha)\left[\|\varphi\|_{E_{\alpha}}+\|\psi\|_{E_{\alpha}}+\max _{1 \leq k \leq N-1}\left\|f_{k}\right\|_{E_{\alpha}}\right]
$$

Theorem 16 For the solution of difference schemes (69) and (70), the following almost coercive stability estimate holds:

$$
\begin{aligned}
& \max _{1 \leq k \leq N-1}\left\|\frac{1}{\tau^{2}}\left(u_{k+1}^{h}-2 u_{k}^{h}+u_{k-1}^{h}\right)\right\|_{\dot{C}_{h}} \\
& +h^{-2 m} \sum_{2 m \leq|p| \leq S}\left\|\Delta_{1-}^{p_{1}} \Delta_{1+}^{p_{2}} \cdots \Delta_{(n-1)-}^{p_{2 n-3}} \Delta_{(n-1)+}^{p_{2 n-2}} u^{h}\right\|_{\dot{C}_{h}}+h^{-2 m} \sum_{\substack{2 m \leq r+s \leq S \\
s \leq m}}\left\|\Delta_{+}^{r} \Delta_{-}^{s} u^{h}\right\|_{\dot{C}_{h}} \\
& \leq M \ln \frac{1}{h}\left[h^{-2 m} \sum_{2 m \leq|p| \leq S}\left\|\Delta_{1-}^{p_{1}} \Delta_{1+}^{p_{2}} \cdots \Delta_{(n-1)-}^{p_{2 n-3}} \Delta_{(n-1)+}^{p_{2 n-2}} \varphi^{h}\right\|_{\dot{C}_{h}}\right. \\
& +h^{-2 m} \sum_{\substack{2 m \leq r+s \leq S \\
s \leq m}}\left\|\Delta_{+}^{r} \Delta_{-}^{s} \varphi^{h}\right\|_{\dot{C}_{h}}+h^{-2 m} \sum_{2 m \leq|p| \leq S}\left\|\Delta_{1-}^{p_{1}} \Delta_{1+}^{p_{2}} \cdots \Delta_{(n-1)-}^{p_{2 n-3}} \Delta_{(n-1)+}^{p_{2 n-2}} \psi^{h}\right\|_{\dot{C}_{h}} \\
& \left.+h^{-2 m} \sum_{\substack{2 m \leq r+s \leq S \\
s \leq m}}\left\|\Delta_{+}^{r} \Delta_{-}^{s} \psi^{h}\right\|_{\dot{C}_{h}}\right]+M \ln \frac{1}{\tau+h} \max _{1 \leq k \leq N-1}\left\|f_{k}^{h}\right\|_{\dot{C}_{h}}
\end{aligned}
$$

The proof of Theorem 16 is based on Theorem 2, on the positivity of an elliptic difference operator $A_{h}^{x}$ in the Banach space $\dot{C}_{h}$, on the estimate

$$
\min \left\{\ln \frac{1}{\tau}, 1+\left|\ln \left\|A_{h}^{x}\right\|_{\dot{C}_{h} \rightarrow \dot{C}_{h}}\right|\right\} \leq M \ln \frac{1}{\tau+h},
$$

and on the following theorem on the almost coercive stability of difference schemes (69) and (70) and on Theorem 7 on the almost coercive stability of elliptic difference problem (66).

Theorem 17 [3] Let A be a positive operator in a Banach space E. Then, for the solution of difference schemes (69) and (70), the following almost coercive stability inequality holds:

$$
\begin{aligned}
& \max _{1 \leq k \leq N-1}\left\|\frac{1}{\tau^{2}}\left(u_{k+1}-2 u_{k}+u_{k-1}\right)\right\|_{E}+\max _{0 \leq k \leq N}\left\|A u_{k}\right\|_{E} \\
& \leq M \min \left\{\ln \frac{1}{\tau}, 1+\left|\ln \|A\|_{E \rightarrow E}\right|\right\} \max _{1 \leq k \leq N-1}\left\|f_{k}\right\|_{E} .
\end{aligned}
$$

Theorem 18 Let $0<2 m \alpha<1$. Then, the solution of the difference schemes (69) and (70) satisfies the following coercive stability estimate:

$$
\begin{aligned}
\max _{1 \leq k \leq N-1}\left\|\frac{1}{\tau^{2}}\left(u_{k+1}^{h}-2 u_{k}^{h}+u_{k-1}^{h}\right)\right\|_{\dot{C}_{h}^{2 m \alpha}} \\
+h^{-2 m} \sum_{2 m \leq|p| \leq S}\left\|\Delta_{1-}^{p_{1}} \Delta_{1+}^{p_{2}} \cdots \Delta_{(n-1)-}^{p_{2 n-3}} \Delta_{(n-1)+}^{p_{2 n-2}} u^{h}\right\|_{\dot{C}_{h}^{2 m \alpha}}
\end{aligned}
$$




$$
\begin{aligned}
& +h^{-2 m} \sum_{2 m \leq r+s \leq S}\left\|\Delta_{+}^{r} \Delta_{-}^{s} u^{h}\right\|_{\dot{C}_{h}^{2 m \alpha}} \\
\leq & M(\alpha)\left[h^{-2 m} \sum_{2 m \leq|p| \leq S}\left\|\Delta_{1-}^{p_{1}} \Delta_{1+}^{p_{2}} \cdots \Delta_{(n-1)-}^{p_{2 n-3}} \Delta_{(n-1)+}^{p_{2 n-2}} \varphi^{h}\right\|_{\dot{C}_{h}^{2 m \alpha}}\right. \\
+ & h^{-2 m} \sum_{2 m \leq r+s \leq S}\left\|\Delta_{+}^{r} \Delta_{-}^{s} \varphi^{h}\right\|_{\dot{C}_{h}^{2 m \alpha}} \\
+ & h^{-2 m} \sum_{\substack{s \leq m \\
2 m \leq|p| \leq S}}\left\|\Delta_{1-}^{p_{1}} \Delta_{1+}^{p_{2}} \cdots \Delta_{(n-1)-}^{p_{2 n-3}} \Delta_{(n-1)+}^{p_{2 n-2}} \psi^{h}\right\|_{\dot{C}_{h}^{2 m \alpha}} \\
+ & \left.h^{-2 m} \sum_{\substack{2 m \leq r+s \leq S \\
s \leq m}}\left\|\Delta_{+}^{r} \Delta_{-}^{s} \psi^{h}\right\|_{\dot{C}_{h}^{2 m \alpha}}\right]+M(\alpha) \max _{1 \leq k \leq N-1}\left\|f_{k}^{h}\right\|_{\dot{C}_{h}^{2 m \alpha}} .
\end{aligned}
$$

The proof of Theorem 18 is based on Theorem 3, on the structure of the fractional spaces $E_{\alpha}\left(\dot{C}_{h}, A_{h}^{x}\right)$, on Theorem 11, on the structure of the fractional space $E_{\alpha}^{\prime}=E_{\alpha}\left(E, A^{1 / 2}\right)$, on Theorem 2, on the positivity of an elliptic difference operator $A_{h}^{x}$ in the Banach space $\dot{C}_{h}$, and on the following theorem on the coercive stability of difference schemes (69) and (70) and on Theorem 13 on the coercive stability of elliptic difference problem (66).

Theorem 19 [2] Let A be a strongly positive operator in a Banach space E. Then, for the solution of difference schemes (69) and (70), the following coercive stability inequality:

$$
\max _{1 \leq k \leq N-1}\left\|\frac{1}{\tau^{2}}\left(u_{k+1}-2 u_{k}+u_{k-1}\right)\right\|_{E_{\alpha}}+\max _{0 \leq k \leq N}\left\|A u_{k}\right\|_{E_{\alpha}} \leq M(\alpha) \max _{1 \leq k \leq N-1}\left\|f_{k}\right\|_{E_{\alpha}}
$$

is valid.

\section{Conclusion}

In the present article, the structure of the fractional spaces $E_{\alpha}\left(\dot{C}\left(\mathbb{R}_{h n}^{+}\right), A_{h}^{x}\right)$ generated by the multi-dimensional elliptic difference operator $A_{h}^{x}$ is investigated.

\section{Competing interests}

The authors declare that they have no competing interests.

Authors' contributions

All authors read and approved the final manuscript.

\section{Author details}

'Department of Elementary Mathematics Education, Fatih University, Buyukcekmece, Istanbul, 34500, Turkey. ${ }^{2}$ Department of Mathematics, ITTU, Ashgabat, Turkmenistan. ${ }^{3}$ Department of Mathematics, Fatih University, Buyukcekmece, Istanbul, 34500, Turkey.

\section{Acknowledgements}

The authors would like to thank Prof. Pavel Sobolevskii (Universidade Federal do Ceará, Brasil). The second author would also like to thank The Scientific and Technological Research Council of Turkey (TUBiTAK) for the financial support.

Received: 24 August 2015 Accepted: 3 December 2015 Published online: 30 December 2015

\section{References}

1. Sobolevskii, PE: A new method of summation of Fourier series converging in C-norm. Semigroup Forum 71, 289-300 (2005)

2. Ashyralyev, A, Sobolevskii, PE: Well-Posedness of Parabolic Difference Equations Operator Theory: Advances and Applications. Birkhäuser, Basel (1994)

3. Ashyralyev, A, Sobolevskii, PE: New Difference Schemes for Partial Differential Equations. Birkhäuser, Basel (2012) 
4. Stewart, HB: Generation of analytic semigroups by strongly elliptic operators under general boundary conditions. Trans. Am. Math. Soc. 259, 299-310 (1980)

5. Solomyak, MZ: Estimation of norm of the resolvent of elliptic operator in spaces $L_{p}$. Usp. Mat. Nauk 15(6), 141-148 (1960)

6. Sobolevskii, PE: The coercive solvability of difference equations. Dokl. Akad. Nauk SSSR 201(5), 1063-1066 (1971)

7. Alibekov, KA: Investigations in $C$ and $L_{p}$ of difference schemes of high order accuracy for approximate solutions of multi-dimensional parabolic boundary value problems. Dissertation, Voronezh State University, Voronezh (1978)

8. Simirnitskii, YA, Sobolevskii, PE: Positivity of multi-dimensional difference operators in the C-norm. Usp. Mat. Nauk 36(4), 202-203 (1981) (Russian)

9. Simirnitskii, YA: Positivity of difference elliptic operators. PhD thesis, Voronezh State University, Voronezh (1983) (Russian)

10. Triebel, H: Interpolation Theory, Function Spaces, Differential Operators. North-Holland, Amsterdam (1978)

11. Danelich, SI: Positive difference operators in $\mathbb{R}_{h 1}$. Voronezh. Gosud. Univ., Deposited VINITI 3.18.1987, vol. 1936-B87, pp. 13 (1987) (Russian)

12. Danelich, SI: Fractional powers of positive difference operators. Dissertation, Voronezh State University, Voronezh (1989)

13. Ashyralyev, A, Akturk, S, Sozen, Y: The structure of fractional spaces generated by a two-dimensional elliptic differential operator and its applications. Bound. Value Probl. 2014, 3 (2014)

14. Ashyralyev, A, Agirseven, D: Well-posedness of delay parabolic difference equations. Adv. Differ. Equ. 2014, 18 (2014)

15. Ashyralyev, A, Nalbant, N, Sozen, Y: Structure of fractional spaces generated by second order difference operators. J. Franklin Inst. 351(2), 713-731 (2014)

16. Ashyralyev, A: Method of positive operators of investigations of the high order of accuracy difference schemes for parabolic and elliptic equations. Dissertation, Inst. of Math. of Acad. Sci., Kiev (1991)

17. Neginskii, BA, Sobolevskii, PE: Difference analogue of theorem on inclosure an interpolation inequalities. In: Proceedings of Faculty of Math., Voronezh State University, vol. 1, pp. $72-81$ (1970) (Russian)

18. Bazarov, MA: On the structure of fractional spaces. In: Proceedings of the XXVII All-Union Scientific Student Conference "The Student and Scientific-Technological Progress", pp. 3-7. Novosibirsk. Gos. Univ., Novosibirsk (1989) (Russian)

19. Ashyralyev, A, Sobolevskii, PE: The linear operator interpolation theory and the stability of the difference schemes. Dokl. Akad. Nauk SSSR 275(6), 1289-1291 (1984)

20. Sobolevskii, PE: Some properties of the solutions of differential equations in fractional spaces. Trudy Nauchn.-Issled. Inst. Mat. Voronezh. Gos. Univ. 74, 68-76 (1975) (Russian)

21. Solomyak, MZ: Analytic semigroups generated by elliptic operator in spaces $L_{p}$. Dokl. Akad. Nauk SSSR 127(1), 37-39 (1959)

22. Stewart, HB: Generation of analytic semigroups by strongly elliptic operators. Trans. Am. Math. Soc. 190, 141-162 (1974)

23. Agmon, S: Lectures on Elliptic Boundary Value Problems. Van Nostrand, Princeton (1965)

24. Agmon, S, Nirenberg, L: Properties of solutions of ordinary differential equations in Banach spaces. Commun. Pure Appl. Math. 20, 121-239 (1963)

25. Agmon, S, Douglis, A, Nirenberg, L: Estimates near the boundary for solutions of elliptic partial differential equations satisfying general boundary conditions I. Commun. Pure Appl. Math. 12, 623-727 (1959)

26. Agmon, S, Douglis, A, Nirenberg, L: Estimates near the boundary for solutions of elliptic partial differential equations satisfying general boundary conditions II. Commun. Pure Appl. Math. 17, 35-92 (1964)

27. Sobolevskii, PE: Well-posedness of difference elliptic equation. Discrete Dyn. Nat. Soc. 1(3), 213-219 (1997)

28. Ashyralyev, A: Well-posedness of the difference schemes for elliptic equations in spaces. Appl. Math. Lett. 22(3), 390-395 (2009)

29. Ashyralyev, A, Sobolevskii, PE: Difference schemes of the high order of accuracy for parabolic equations with variable coefficients. Dokl. Akad. Nauk Ukrainian SSR, Ser. A Fiz.-Mat. Tech. Sci. 6, 3-7 (1988) (Russian)

30. Semenova, GE: Positivity of elliptic difference operators and its applications. In: ICAAM 2012. AIP Conference Proceedings, vol. 1470, pp. 232-235 (2012)

\section{Submit your manuscript to a SpringerOpen ${ }^{\odot}$ journal and benefit from:}

- Convenient online submission

Rigorous peer review

- Immediate publication on acceptance

- Open access: articles freely available online

- High visibility within the field

- Retaining the copyright to your article 